\title{
Heterosis and epistasis for teat number and fluctuating asymmetry in crosses between Jiaxing and Iberian pigs
}

\author{
A Fernández, M Toro, C Rodríguez and L Silió \\ Departamento de Mejora Genética Animal, INIA, Carretera La Coruña km. 7, Madrid 28040, Spain
}

\begin{abstract}
In the framework of Wright's view of evolution, long-separate breeds of domestic animals could establish different adaptive epistatic genetic complexes that could be destroyed in crossbred animals by recombination. The objective of this study was to evaluate heterosis and recombination effects in a crossing experiment involving two distinct European and Asian breeds (Iberian and Jiaxing) in the $\mathrm{F} 1$ and two successive backcrosses to the Iberian line. Teat number (TN) was recorded in the right and left sides of piglets and analysed by fitting a mixed linear model including the Dickerson's crossbreeding parameters. TN in pigs is a discontinuous and often canalised trait presenting bilateral symmetry. The minor differences between sides make this trait a good candidate to evaluate fluctuating asymmetry (FA) and developmental instability. For TN, the posterior means
\end{abstract}

and standard deviations (SD) of the heritability and of the relative contribution of common litter environmental effect to variance were $0.248(0.028)$ and $0.057(0.019)$, respectively. The respective values of the difference between breeds, heterosis and recombination effect were 9.990 (0.411), $-0.506(0.196)$ and $0.684(0.232)$. For FA, the posterior means and SDs of the heritability and of the relative contribution of common litter environmental effect to variance were $0.023(0.005)$ and $0.014(0.005)$, respectively. Another significant genetic effect was a recombination effect of 0.773 (0.117). These results confirm that the rupture by recombination of coadapted genomes decreases developmental stability in domestic pigs.

Heredity (2004) 93, 222-227, advance online publication, 2 June 2004; doi:10.1038/sj.hdy.6800498

Keywords: Iberian pig; teat number; fluctuating asymmetry; heterosis; recombination effect

\section{Introduction}

One of the lines of evidence that led to Wright's view of evolution, known as the shifting balance process, was his study of the formation of the Shorthorn breed (Wright, 1978). The domestication process provided the conditions for the shifting balance to occur because small populations captured from the wild were bred many times and in many places. Those more productive and adapted to human management probably replaced those less successful. As Wright believed that epistasis makes a major contribution to fitness, his theory provides a way for explaining the fixation of favourable epistatic gene complexes. It seems reasonable that long-separate breeds have undergone independent evolution via artificial selection and genetic drift, establishing different adaptive epistatic genetic complexes that could be destroyed by recombination. The crosses between temperate dairy breeds and zebu local breeds in the tropics are a paradigmatic example. In almost all cases the F1 was superior to the parental breeds, but the F2 generation fails to meet the expectations based on additive and dominance effects, and this is generally attributed to the break-up of epistatic complexes (see, Rutledge, 2001, for a review).

Correspondence: $M$ Toro, Departamento de Mejora Genética Animal, INIA, Carretera La Coruña km. 7, Madrid 28040, Spain.

E-mail: toro@inia.es

Received 11 November 2002; accepted 6 April 2004; published online 2 June 2004
The objective of this study is to evaluate heterosis and recombination effects in a crossing experiment between pigs of two distinct European and Asian breeds: Iberian and Jiaxing. The history and structure of these breeds meet the requirements of Wright's scenario for fixing adaptive epistatic complexes. The two breeds were produced from independent domestication processes, and have been largely isolated and selected for divergent purposes. The Iberian is a breed of low prolific autochthonous pigs, which has been maintained for centuries in large areas of the south-western Iberian Peninsula and constitutes the largest of the surviving populations of the Mediterranean type (Dobao et al, 1988). The Jiaxing, as other Chinese breeds of the Taihu group, is famous for being highly prolific, whereas other characteristics like rusticity, slow growth, fat carcasses and reputed meat quality are shared with the Iberian pig (Legault and Caritez, 1983).

For this purpose, we use line-cross analysis of number of teats and their asymmetry. Teat number (TN) presents bilateral symmetry, with only minor differences between the two sides. Besides, TN is a canalised trait, at least in Iberian pigs (Toro et al, 1986), that is, it presents an excess of values close to the mean. It is an heritable trait with moderate heritability (Toro et al, 1986; Béjar et al, 1993; Zhang et al, 2000). Fluctuating asymmetry (FA), defined as small random deviations from identical expression across an axis of symmetry, is one of the three types of asymmetry described. It is commonly interpreted as a measurable expression of developmental instability 
(Palmer and Strobeck, 1986) with a genetic basis, that is, character, population and taxon specific (Clarke, 1998). Although the proportion of FA variation under additive genetic control is a controversial topic (Møller and Swaddle, 1997), the general consensus is that the heritability of FA is usually quite low in magnitude or even zero (Whitlock and Fowler, 1997; Fuller and Houle, 2003). However, nonadditive genetic effects, intralocus (dominance) and interloci (epistasis) interactions, are commonly invoked to explain FA variation. In this sense, significant dominant and epistatic effects for FA of mandible size have been found in mice (Leamy et al, 1998, 2002).

The specific goals of the study are as follows: (a) to characterise the type of asymmetry in TN in pigs, (b) to estimate the heritability of TN and FA, (c) to estimate the genetic covariance between both traits and (d) to estimate heterotic and epistatic effects on both traits.

\section{Material and methods}

\section{Populations}

The experiment involved Chinese pigs from the Jiaxing breed (P1) and Iberian pigs from the Torbiscal strain (P2). To produce the F1 animals, semen from five Jiaxing boars was used to inseminate 17 Torbiscal sows. Later, generations were obtained from two successive backcrosses to Torbiscal sires: P2 $\times$ F1 (B2) and P2 $\times$ B2 . The total number of pigs with available records was 8779 , from both sexes born in 1119 litters, and includes not only the animals involved in the experiment but also contemporaneous purebred Torbiscal pigs. The pedigree consisted of 9966 animal-sire-dam entries. The number of teats was scored in both the right (R) and the left (L) side when piglets were 21 days old. The values of signed $(\mathrm{R}-\mathrm{L})$ and unsigned $|\mathrm{R}-\mathrm{L}|$ difference between the right and the left sides were calculated for all the individuals. These differences account, respectively, for the directional asymmetry (DA) and FA of the TN.

\section{Statistical methods}

The following univariate mixed linear model was used to estimate genetic effects for the total number of teats and the FA

$$
y=X \beta+Z_{1} u+Z_{2} c+e
$$

where $y$ is the vector of observations; $X, Z_{1}$ and $Z_{2}$ are known incidence matrices relating location parameters $(\beta)$, and random effects ( $u$ and $c$ ), to $y ; u$ is the vector of additive genetic effects; $c$ is the vector of common litter environmental effects; $e$ the vector of random residuals; and $\beta$ is the vector of systematic effects, including sex (two levels), batch (23 levels) and three parameters, representing genetic effects expressed in each line or cross according to Dickerson (1969).

The Dickerson's model (1969) is framed in terms of: (1) additive difference between breeds (P1-P2); (2) heterosis $(H)$, defined as $H=\mathrm{F} 1-(\mathrm{P} 1+\mathrm{P} 2) / 2$; and (3) recombination effect, defined as $R=\mathrm{F} 2-H$. The coefficients that show the proportion of these effects expressed in the purebred and crossbred animals are usually referred as $p$ (the proportion of genes of breed P1), the coefficient of racial heterozygosity $h$ (the probability that two alleles, taken at random in one locus, are from different breeds)
Table 1 Coefficients of the genetic effects for the parameterisation of the Dickerson's model

\begin{tabular}{lccc}
\hline & $P 1-P 2$ & $\mathrm{H}$ & $\mathrm{R}$ \\
\cline { 2 - 4 } Line or cross & $\mathrm{p}$ & $\mathrm{h}$ & $\mathrm{r}$ \\
\hline Jiaxing (P1) & 1 & 0 & 0 \\
Iberian (P2) & 0 & 0 & 0 \\
F1 $($ P1 $\times$ P2) & $1 / 2$ & 1 & 0 \\
B2 $($ P2 $\times$ F1) & $1 / 4$ & $1 / 2$ & $1 / 4$ \\
P2 $\times$ B2 & $1 / 8$ & $1 / 4$ & $3 / 16$ \\
\hline
\end{tabular}

and the coefficient of recombination effect $r$ (the average fraction of pairs of loci in gametes from both parents that are expected to be nonparental combinations). Their values in the populations of the present experiment are indicated in Table 1 . It should be noted that, given that records from an F2 generation were unavailable, the estimates of recombination effects were obtained only from the two successive backcrosses.

Data were analysed using two different approaches. First, variance components $\left(\sigma_{u}^{2}, \sigma_{c}^{2}, \sigma_{e}^{2}\right)$ were estimated by standard restricted maximum likelihood (REML) and their statistical significance was tested by comparing nested models with the same systematic-effects structure using the restricted likelihood ratio (LR) statistic. The distribution of the LR statistic is a 50:50 mixture of $\chi_{(1)}^{2}$ and $\chi_{(0)}^{2}$ (Stram and Lee, 1994). As the $P$-value associated with $\chi_{(0)}^{2}$ equals zero, the overall significance levels equals the $P$-value associated with $\chi_{(1)}^{2}$ divided by two. Unsigned individual FA values are typically half-normally distributed. Although deviations from normality affect statistical testing of the variance components, the estimates remain unbiased (Meyer, 1985). Best linear unbiased estimates of fixed effects were obtained for both traits assuming that the REML estimates are the true variances.

A Bayesian analysis was performed using Gibbs sampling techniques (Wang et al, 1994) to obtain inferences on the parameters of interest: variance components, heritability $\left(h^{2}=\sigma_{u}^{2} / \sigma_{P}^{2}\right)$, coefficient of common litter environmental effects $\left(c^{2}=\sigma_{c}^{2} / \sigma_{P}^{2}\right)$ and Dickerson's crossbreeding parameters, based on their marginal posterior distributions. An additional Bayesian analysis was performed for TN and FA fitting a bivariate model (Rodriguez et al, 1996) with the same structure of effects. For each analysis, a single Gibbs chain of 1010000 samples was obtained. The convergence was assessed by the double chain method (Garcia Cortés et al, 1998). Flat priors were used for all the parameters. The usual statistics of location (posterior mean, mode and median) and dispersion (posterior standard deviation (SD) and 95\% highest posterior density interval) were calculated from saved samples.

\section{Results}

The number of individuals scored in the pure lines and crosses, the total number of teats in both sides and several statistics for this trait (SD, skewness and kurtosis) are shown in Table 2. TN ranged from 9 to 21 across individuals in the whole data set. The average number of teats in the Jiaxing is almost double that of the Iberian pigs and the crosses show intermediate values. The data 
Table 2 Number of animals $(n)$, mean, SD, coefficients of skewness $\left(g_{1}\right)$ and kurtosis $\left(g_{2}\right)$ of TN, signed $(\mathrm{R}-\mathrm{L})$ and unsigned $|\mathrm{R}-\mathrm{L}|$ differences between right (R) and left $(\mathrm{L})$ sides for TN in Jiaxing and Iberian pigs and their crosses

\begin{tabular}{|c|c|c|c|c|c|c|c|c|c|c|c|}
\hline \multirow[t]{2}{*}{ Line or cross } & \multirow[t]{2}{*}{$\mathrm{n}$} & \multicolumn{4}{|c|}{$T N$} & \multicolumn{4}{|c|}{$(R-L)$} & \multicolumn{2}{|c|}{$|R-L|$} \\
\hline & & Mean & $S D$ & $g_{1}$ & $g_{2}$ & Mean & $S D$ & $g_{1}$ & $g_{2}$ & Mean & $S D$ \\
\hline Jiaxing (P1) & 5 & 19.80 & 1.10 & - & - & 0.20 & 0.45 & - & - & 0.20 & 0.45 \\
\hline Iberian (P2) & 5713 & 10.24 & 0.54 & $2.11^{* * *}$ & $4.81^{* * *}$ & -0.01 & 0.42 & -0.04 & $3.78^{* * * *}$ & 0.17 & 0.38 \\
\hline $\mathrm{F} 1(\mathrm{P} 1 \times \mathrm{P} 2)$ & 98 & 14.54 & 1.04 & -0.39 & 0.05 & 0.11 & 0.67 & $0.49^{*}$ & 0.75 & 0.40 & 0.55 \\
\hline $\mathrm{B} 2(\mathrm{P} 2 \times \mathrm{F} 1)$ & 2230 & 12.60 & 1.15 & -0.02 & $-0.23^{*}$ & 0.05 & 0.68 & 0.06 & $0.76^{* * *}$ & 0.42 & 0.54 \\
\hline $\mathrm{P} 2 \times \mathrm{B} 2$ & 733 & 11.28 & 1.07 & $0.45^{* * *}$ & -0.34 & -0.00 & 0.68 & -0.02 & $0.77^{* * *}$ & 0.41 & 0.55 \\
\hline Total & 8779 & 10.98 & 1.36 & $1.36^{* * *}$ & $1.57^{* * *}$ & 0.01 & 0.53 & $0.09^{* * *}$ & $2.50^{* * *}$ & 0.26 & 0.46 \\
\hline
\end{tabular}

Test significance of $g_{1}$ and $g_{2}:{ }^{* * *} P<0.001 ;{ }^{* *} P<0.01 ;{ }^{*} P<0.05$.

Table 3 Estimated statistics of marginal posterior distributions of genetic parameters and Dickerson's crossbreeding parameters from univariate analysis for total number of teats and FA compared with standard REML estimates

\begin{tabular}{|c|c|c|c|c|c|}
\hline & Mean & Mode & $P S D$ & $95 \% H P D$ & REML estimate \\
\hline \multicolumn{6}{|l|}{$T N$} \\
\hline$h^{2}=\sigma_{u}^{2} / \sigma_{P}^{2}$ & 0.241 & 0.231 & 0.031 & $0.182 / 0.301$ & 0.362 \\
\hline$c^{2}=\sigma_{c}^{2} / \sigma_{P}^{2}$ & 0.026 & 0.026 & 0.006 & $0.014 / 0.038$ & 0.021 \\
\hline Difference between breeds (P1-P2) & 9.444 & 9.463 & 0.481 & $8.487 / 10.372$ & 9.444 \\
\hline Heterosis $(H)$ & -0.525 & -0.536 & 0.195 & $-0.912 /-0.149$ & -0.533 \\
\hline Recombination effect $(R)$ & 0.670 & 0.678 & 0.219 & $0.250 / 1.098$ & 0.682 \\
\hline \multicolumn{6}{|l|}{$F A$} \\
\hline$h^{2}=\sigma_{u}^{2} / \sigma_{P}^{2}$ & 0.042 & 0.041 & 0.011 & $0.022 / 0.062$ & 0.055 \\
\hline$c^{2}=\sigma_{c}^{2} / \sigma_{P}^{2}$ & 0.022 & 0.022 & 0.007 & $0.007 / 0.036$ & 0.013 \\
\hline Difference between breeds (P1-P2) & 0.026 & 0.005 & 0.218 & $-0.404 / 0.450$ & 0.023 \\
\hline Heterosis $(H)$ & 0.149 & 0.147 & 0.113 & $-0.073 / 0.369$ & 0.158 \\
\hline Recombination effect $(R)$ & 0.723 & 0.728 & 0.114 & $0.502 / 0.948$ & 0.724 \\
\hline
\end{tabular}

PSD: posterior standard deviation.

95\% HPD: 95\% highest posterior density interval.

confirm that $\mathrm{TN}$ in Iberian pigs is a canalised trait with small variance and positive kurtosis (Toro et al, 1986). The distribution shows skewness to the right $\left(g_{1}=2.11\right.$; $\left.P<10^{-7}\right)$ and leptokurtosis $\left(g_{2}=4.81 ; P<10^{-7}\right)$, with most of the items close to the mean. Both skewness to the right and positive kurtosis disappear in the crossbred animals. Owing to the low number of available data for purebred Jiaxing animals, the $g_{1}$ and $g_{2}$ statistics were not calculated.

The values of mean, SD and statistics measuring skewness $\left(g_{1}\right)$ and kurtosis $\left(g_{2}\right)$ of $(\mathrm{R}-\mathrm{L})$ differences are shown in Table 2 separately by line or cross as well as for data pooled across genetic types. The signed difference between sides ranged from -3 to +3 . For traits showing FA, the distribution of signed differences (R-L) would have a mean zero and would not exhibit platykurtosis (Palmer and Strobeck, 1986). Departures in these moments can indicate DA or antisymmetry. The total mean of these values did not differ significantly from zero $(t=1.78 ; \mathrm{df}=8778 ; P<0.076)$ and thus DA can be discounted. None of these distributions showed a platykurtic departure from normality and thus no antisymmetry is present. These results allow the differences in TN found between right and left sides to be characterised as FA. The mean and SD of unsigned $|\mathrm{R}-\mathrm{L}|$ differences by line or cross are also shown in Table 2.

The results of the main statistics of marginal posterior distributions of genetic parameters from the univariate analysis of TN and FA are presented in Table 3. For TN, the posterior means and SDs of the additive $\left(\sigma_{u}^{2}\right)$, common litter environmental $\left(\sigma_{c}^{2}\right)$, residual $\left(\sigma_{e}^{2}\right)$ and phenotypic $\left(\sigma_{P}^{2}\right)$ variances were 0.158 (0.022), 0.017 $(0.004), 0.481(0.014)$ and $0.657(0.013)$, respectively. The heritability $\left(h^{2}\right)$ of TN was greater than the proportion of phenotypic variance due to common litter environmental effects $\left(c^{2}\right)$. The comparison of nested models indicated, however, a significant common environmental effect $(\mathrm{LR}=19.59, P<0.002)$. With respect to the effects of Dickerson's crossbreeding parameters on TN, the following point estimates were obtained from the Bayesian univariate analysis: a difference between Jiaxing and Iberian breeds of 9.444 teats, a negative value of heterosis $(H=-0.525)$ and a positive value of the recombination effect $(R=0.670)$. The corresponding 95\% HPD intervals do not include the zero value.

For FA, the means and SDs of $\sigma_{u}^{2}, \sigma_{c}^{2}, \sigma_{e}^{2}$ and $\sigma_{P}^{2}$ were 0.008 (0.002), 0.004 (0.001), $0.188(0.003)$ and $0.200(0.001)$, respectively. The Bayesian posterior interval of $h^{2}$ for FA (Table 3 ) and the value of the corresponding test statistic in REML analysis $\left(\mathrm{LR}=59.0, P<10^{-7}\right)$ indicate a significant additive genetic variance component for this trait. No evidence of a significant common litter environmental effect for FA was found in the REML analysis $(\mathrm{LR}=5.21, P<0.107)$, although the 95\% HPD interval of $c^{2}$ did not include the zero value. With respect to the Dickerson's crossbreeding parameters and according the bounds of their 95\% HPD intervals, the difference between breeds and the heterosis effect on FA were not significantly different from zero. A significant value of the recombination effect of 0.723 was found for this measure of asymmetry (Table 3 ). 
Table 4 Estimated statistics of marginal posterior distributions of genetic parameters (heritability, $h^{2}$, common environmental, $c^{2}$, and correlations) and Dickerson's crossbreeding parameters obtained from bivariate analysis for TN and FA

\begin{tabular}{|c|c|c|c|c|c|}
\hline & Mean & Mode & Median & $P S D$ & $95 \% H P D$ \\
\hline \multicolumn{6}{|l|}{$T N$} \\
\hline$h^{2}=\sigma_{u}^{2} / \sigma_{P}^{2}$ & 0.248 & 0.241 & 0.246 & 0.028 & $0.197 / 0.301$ \\
\hline$c^{2}=\sigma_{c}^{2} / \sigma_{P}^{2}$ & 0.057 & 0.063 & 0.057 & 0.019 & $0.026 / 0.093$ \\
\hline Difference between breeds (P1-P2) & 9.990 & 10.028 & 9.990 & 0.411 & $9.216 / 10.815$ \\
\hline Heterosis $(H)$ & -0.506 & -0.528 & -0.510 & 0.196 & $-0.888 /-0.119$ \\
\hline Recombination effect $(R)$ & 0.684 & 0.717 & 0.685 & 0.232 & $0.227 / 1.135$ \\
\hline \multicolumn{6}{|l|}{$F A$} \\
\hline$h^{2}$ & 0.023 & 0.021 & 0.023 & 0.005 & $0.013 / 0.034$ \\
\hline$c^{2}$ & 0.014 & 0.015 & 0.014 & 0.005 & $0.004 / 0.023$ \\
\hline Difference between breeds (P1-P2) & 0.149 & 0.139 & 0.147 & 0.211 & $-0.251 / 0.570$ \\
\hline Heterosis $(H)$ & 0.118 & 0.127 & 0.122 & 0.112 & $-0.100 / 0.331$ \\
\hline Recombination effect $(R)$ & 0.773 & 0.758 & 0.772 & 0.117 & $0.547 / 1.003$ \\
\hline \multicolumn{6}{|l|}{ Correlations } \\
\hline Genetic $\rho_{G}$ & 0.920 & 0.969 & 0.928 & 0.045 & $0.838 / 0.980$ \\
\hline Common environmental $\rho_{c}$ & 0.010 & 0.016 & 0.010 & 0.043 & $-0.073 / 0.094$ \\
\hline Residual $\rho_{e}$ & 0.189 & 0.189 & 0.190 & 0.016 & $0.158 / 0.221$ \\
\hline Phenotypic $\rho_{P}$ & 0.264 & 0.264 & 0.264 & 0.012 & $0.241 / 0.288$ \\
\hline
\end{tabular}

PSD: posterior standard deviation.

95\% HPD: 95\% highest posterior density interval.

The results of the bivariate analysis for TN and FA are summarised in Table 4 . Differences with the univariate analysis were minor for most point estimates. A high value of genetic correlation between $\mathrm{TN}$ and FA was found. According to the bounds of 95\% HPD intervals, the effects of the Dickerson's crossbreeding parameters on TN and the recombination effect on FA were significantly different from zero.

\section{Discussion}

Both plant and animal breeders know that crossbreeding of two breeds or lines has usually positive effects on the fitness of F1 offspring. The superiority of the performance of F1 over the average parental performance is called heterosis or hybrid vigour. Nevertheless, there are exceptions observed frequently in crosses between different species or distantly related breeds, a phenomenon known as outbreeding depression. In these cases, hybridisation increases heterozygosity and disrupts coadapted gene complexes. Examples range from species of Drosophila, populations of marine copepodes, Daphnia clones and populations of many outbreeding plants even separated by a few tens of meters (see, Lynch and Walsh, 1998, for a review).

Hill (1982) proposed a different parameterisation to that of Dickerson in terms of additive effects of genes $(A)$, dominance effects $(D)$, additive $\times$ additive $(\mathrm{AA})$, additive $\times$ dominance $(\mathrm{AD})$ and dominance $\times$ dominance (DD). The relation between Dickerson and Hill's models is useful to interpret the aforementioned phenomenon. The heterosis, measured as the difference between the F1 and the parental mean, is a function of both dominance and additive $\times$ additive interaction $H=2 D-\mathrm{AA}$ and the recombination effect $(R=\mathrm{F} 2-H)$ is entirely a function of epistatic effects $R=-\mathrm{AA}+2 \mathrm{AD}-4 \mathrm{DD}$. The common explanation for heterosis is the presence of negative recessive alleles in the parental lines whose effects are masked in the F1. With epistasis, the net value of heterosis depends on the AA interaction. The results for $\mathrm{TN}$ in this study show a heterosis value of -0.506 and a recombination effect of 0.684 . To explain sign and size of these values requires a combination of dominant (D) and epistatic (AA, AD and DD) effects. Unfortunately, there are not enough line-cross means to estimate all of the components of Hill's model. In any case, the magnitude of the $H$ and $R$ effects is quite small relative to the average parental value (about $4 \%$ ) and other alternative explanations (maternal effects or imprinting) are also possible. Both maternally and paternally imprinted QTL for TN have been reported in pigs (Hirooka et al, 2001).

The estimated values of Dickerson's parameters for FA indicate a significant recombination effect. However, the difference between breeds and heterosis effects were not significant. The lack of significant heterosis for FA could result from a balance between the opposite effects of dominance (decreasing FA) and disruption of additive $\times$ additive interactions (increasing FA). Previous studies of FA in other organisms revealed either increased, decreased or similar FA in hybrid progenies compared to parental lines (Palmer and Strobeck, 1986; Leary and Allendorf, 1989; Markov and Ricker, 1991; Lu and Bernatchez, 1999). A positive value (0.777) of the recombination effect on FA was obtained in the present study, which is more than three times the average of the FA in the parental lines. It implies that the break-up of parental coadapted gene complexes induces a greater developmental instability.

The REML estimate of heritability for TN exceeds the value of posterior mean obtained in the Bayesian approach. Gianola and Foulley (1990) have pointed out that REML estimates of variance components are only marginal with respect to fixed effects, but not so with respect to other dispersion parameters of the model. However, the Bayesian analysis allows further marginalisation, which is particularly valuable for models with several variance components. This is one of the several advantages attributed to the Bayesian implementation of the mixed linear models (Wang et al, 1994). 
Our study suggests a small although significant value of the heritability for FA (2-5\%) similar to those reviewed by Fuller and Houle (2003). However, this result must be treated cautiously because of the difficulties for testing the nule hypothesis when the posterior distributions present peaks close to bounds of parametric space. Although, in theory, the problem can be rigorously solved by the calculation of Bayes factors, this has a huge computational cost (García Cortés et al, 2001). Hence, our analysis used the LR statistic for comparing nested models (Van Dongen et al, 1999). Another problem arises from the lack of normality of FA, which violates an assumption of the statistical tests. Many authors have suggested the use of Box-Cox transformation, but it is a dubious procedure for half-normal distributions because it could magnify the error variance (Fuller and Houle, 2003). Other alternatives such as the use of threshold models have still not been evaluated for analysing this type of trait.

The genetic correlation between TN and FA was high (above 0.90). High genetic correlations between traits and their FA have been usually reported in empirical studies. This indicates that most of the loci responsible for the genetic variation of FA of a particular trait (TN in this study) are the same as those affecting the mean of that trait (Klingenberg and Nijhout, 1999).

The Asian and European types of pigs were domesticated independently and the time of genetic divergence between their common ancestors can be estimated by mtDNA studies (Giuffra et al, 2000). Sequencing of Dloop region and cytochrome $b$ in Iberian pigs has established their European origin. Also, unlike other European breeds there does not seem to be detectable introgression from Asian types (Alves et al, 2003). Therefore, it could be assumed that ancestors of the actual Jiaxing and Iberian pigs have been isolated and evolved independently for about 600000 years (Alves et al, 2003). According to Wright's theory, this long period of genetic isolation and the differing environmental and management conditions would lead to the fixation of distinct sets of genes with favourable interactions. Meiotic recombination via successive backcrossing can destroy some of these interactions, diminishing the TN and increasing the developmental instability, as the results of this study confirm.

\section{Acknowledgements}

Semen dosis of Jiaxing boars were gently provided by INRA (Domaine Le Magneraud, France). We thank the help of JC Caritez, MT Dobao, J Rodrigáñez and the staff of the CIA Dehesón del Encinar. Financial support of the INIA Grant RZ01-028 is acknowledged.

\section{References}

Alves E, Óvilo C, Rodriguez C, Silió L (2003). Mitochondrial DNA sequence variation and phlylogenetic relationships of Iberian pigs and other domestic and wild pig populations. Anim Genet 34: 319-324.

Béjar F, Rodriguez MC, Toro M (1993). Estimation of genetic trends for weaning weight and teat number in Iberian pigs using mixed-model methodology. Livest Prod Sci 33: 239-251.

Clarke GM (1998). The genetic basis of developmental stability. IV. Individual and population asymmetry parameters. Heredity 80: 553-561.
Dickerson GE (1969). Experimental approaches in utilizing breed resources. Anim Breed Abstr 37: 191-202.

Dobao MT, Rodrigañez J, Silió L, Toro MA (1988). Iberian pig production in Spain. Pig News Inform 9: 277-282.

Fuller RC, Houle D (2003). Inheritance of developmental instability. In: Polak $\mathrm{M}$ (eds) Developmental Instability: Causes and Consequences. Oxford University Press: Oxford, pp 157-184.

García Cortés L, Cabrillo C, Moreno C, Varona L (2001). Hypothesis testing for the genetic background of quantitative traits. Genet Sel Evol 33: 3-16.

Garcia Cortés L, Rico M, Groeneveld E (1998). Using coupling with the Gibbs sampler to assess convergence in animals models. J Anim Sci 76: 441-447.

Gianola D, Foulley JL (1990). Variance estimation from integrated likelihood (VEIL). Genet Sel Evol 22: 403-417.

Giuffra E, Kijas JMH, Armager V, Carlborg O, Leon JT, Andersson L (2000). The origin of the domesticated pig: independent domestication and subsequent introgression. Genetics 154: 1875-1891.

Hill WG (1982). Dominance and epistasis as components of heterosis. Z Tierzuchtg Zuchtgsbiol 99: 161-168.

Hirooka H, de Koning DJ, Harlizius B, van Arendonk JAM, Rattink AP, Groenen MAM et al (2001). A whole-genome scan for quantitative trait loci affecting teat number in pigs. J Anim Sci 79: 2320-2326.

Klingenberg CP, Nijhout HF (1999). Genetics of fluctuating asymmetry: a developmental model of developmental instability. Evolution 53: 358-375.

Leamy LJ, Routman EJ, Cheverud J (1998). Quantitative trait loci for fluctuating asymmetry of discrete skeletal charactersin mice. Heredity 80: 509-518.

Leamy LJ, Routman EJ, Cheverud J (2002). An epistatic genetic basis for fluctuating asymmetry of mandible size in mice. Evolution 56: 642-653.

Leary RF, Allendorf FW (1989). Fluctuating asymmetry as an indicator of stress in conservation biology. Trends Ecol Evol 4: 214-217.

Legault C, Caritez JC (1983). L'expérimentation sur le porc chinois en France. I. Performances de reproduction en race pure et en croisement. Genet Sel Evol 15: 225-240.

Lu G, Bernatchez L (1999). A study of fluctuating asymmetry in hybrids of dwarf and normal lake whitefish ecotypes (Coregonus clupeaformis) from different glacial races. Heredity 83: 742-747.

Lynch M, Walsh B (1998). Genetics and Analysis of Quantitative Traits. Sinauer Associates: Sunderland.

Markov TA, Ricker JP (1991). The developmental stability in hybrids between the sibling species pair, Drosophila melanogaster and Drosophila simulans. Genetica 84: 115-121.

Meyer K (1985). Maximum likelihood estimation of variance components for a multivariate mixed model with equal design matrices. Biometrics 41: 153-165.

Møller AP, Swaddle JP (1997). Asymmetry, Development Stability and Evolution. Oxford Universtiy Press: Oxford.

Palmer AR, Strobeck C (1986). Fluctuating asymmetry: measurements, analysis, patterns. Annu Rev Ecol Syst 17: 391-421.

Rodriguez MC, Toro M, Silió L (1996). Selection on lean growth in a nucleus of Landrace pigs: an analysis using Gibbs sampling. Anim Sci 63: 243-253.

Rutledge JJ (2001). Greek temples, tropical kine and recombination load. Livest Prod Sci 68: 171-179.

Stram DO, Lee JW (1994). Variance components testing in the longitudinal mixed effects model. Biometrics 50: 1171-1177.

Toro MA, Dobao MT, Rodrigáñez J, Silió L (1986). Heritability of a canalized trait: teat number in Iberian pigs. Genet Sel Evol 18: $173-184$.

Van Dongen S, Moleneberghs G, Matthysen E (1999). The statistical analysis of fluctuating asymmetry: REML estimation of a mixed regression model. J Evol Biol 12: 94-102. 
Wang CS, Rutledge JJ, Gianola D (1994). Bayesian analysis in mixed linear models via Gibbs sampling with an application to litter size in Iberian pigs. Genet Sel Evol 26: 91-115.

Whitlock M, Fowler K (1997). The instability of studies of instability. J Evol Biol 10: 63-67.
Wright S (1978). The relation of livestock breeding to theories of evolution. I Anim Sci 46: 1192-1200.

Zhang S, Bidanel JP, Burlot T, Legault C, Naveau J (2000). Genetic parameters and genetic trends in the Chinese $\times$ European Tiameslan I. Genetic parameters. Genet Sel Evol 32 $41-56$. 